\title{
Fiber based hydrophones for ultra-high energy neutrino detection
}

\author{
E. J. Buis ${ }^{* a}$, E. J. J Doppenberg ${ }^{a}$, D. van Eijk ${ }^{a}$, R. Lahmann ${ }^{b}$, R. A. Nieuwland ${ }^{a}$ and \\ P. M Toet ${ }^{a}$ \\ ${ }^{a}$ TNO Technical Sciences, P.O. Box 155, 2600 AD Delft, The Netherlands \\ ${ }^{b}$ Erlangen Centre for Astroparticle Physics, University of Erlangen, Erwin-Rommel-Str. 1, 91058 \\ Erlangen, Germany \\ E-mail: ernst-jan.buis@tno.nl
}

\begin{abstract}
It is a well studied process $[1,2]$ that energy deposition of cosmic ray particles in water that generate thermo-acoustic signals. Hydrophones of sufficient sensitivity could measure this signal and provide a means of detecting ultra-high energetic cosmic neutrinos. We investigate optical fiberbased hydrophone technology that could potentially have several advantages over conventional hydrophones based on piezo ceramics. Optical fibers offer a natural way to create a distributed sensing system in which several sensors are attached to a single fiber. The detection system in this case would consist of several sensors, an erbium doped fiber and an interferometric interogator. Next to the advantage of having multiple sensors on a single fiber, this technology has a low power consumption, small size and low weight. Maybe even more important, fiber optics technology provides a cost-effective and straightforward way to implement a large number of hydrophones. An investigation has been carried out to study the feasibility of using fiber based hydrophones in an application for cosmic ray particle detection. We find that the hydrophone technology as explained in this paper offers the proper sensitivity as required to detect low signals orginating from the cosmic rays.
\end{abstract}

International Conference on Technology and Instrumentation in Particle Physics Amsterdam, the Netherlands

June, 2-6 2014

${ }^{*}$ Speaker. 


\section{Introduction}

Results as recently presented by the IceCube collaboration have shown a significant excess of neutrinos (with energies above $60 \mathrm{TeV}$ ) above expected atmospheric muon and neutrino background [3]. These exiting results mark the birth of neutrino astronomy and may lead to the access of a new and rich field of particle astronomy. High energetic neutrinos can provide a unique view on distant and violent astrophysical objects such as gamma-ray bursts and even exotic phenomena, such as Z-bursts. While the universe becomes opaque to high energetic photons and protons due to the increase of the interaction cross-section with the microwave background radiation, neutrinos can travel relatively undisturbed over large distances.

High energy neutrino in the $\mathrm{PeV}$ and $\mathrm{EeV}$ rang may also provide crucial information about the nature of the GZK cut-off [4]. The upper limit of energy spectrum of hadronic cosmic rays has been predicted over 50 years ago and confirmed with measurements of the HiRes and Pierre Auger collaborations [5, 6]. Still, the debate on the nature and origin of the cosmic rays with the highest observed energy is not settled. High energetic cosmic rays may consist of protons, heavy ions or mixtures. A flux measurement of neutrinos that are produced when high energetic cosmic rays interact with the cosmic microwave background could solve this puzzle.

To study the high energy end of the cosmic neutrino spectrum it is required to construct large neutrino telescopes as the flux of these particles is extremely low. In [7] the expected flux and the required sensitivity is discussed in view of the IceCube results. In this paper it is motivated that a telescope of at least $100 \mathrm{~km}^{2}$ is required to extend the measurements of IceCube to a higher energy regime and to contribute to the GZK discussion. Such a neutrino telescope could be formed by a large sensor network equipped with a large number of hydrophones.

\section{Acoustic cosmic neutrino detection}

\subsection{Acoustic signal}

The mechanism of acoustic detection of energetic particles has been investigated already in the 1960's with pioneering work carried out by Askaryan et al $[8,9]$. Measurements were carried out at proton accelerators to investigate the acoustic signals induced by the energy loss of energetic particles in water.

In the case of neutrino detection, the particle detection is detected indirectly after a neutrino has interacted through the neutral and charged current weak interactions which induce a shower of secondary particles in water. The particle shower forms a long line acoustic source of several $\mathrm{cm}$ in radius and about several tens meters long. This line source gives rise to a distinct signal of which an example is shown in figure 1. The shape of the signal is a typical bipolar pulse.

The relative power spectrum as shown in figure 1 (b) shows that the expected frequency range is found between 1 and $50 \mathrm{kHz}$, with a peak power around $10 \mathrm{kHz}$. Note that the peak in the power spectrum is closely related to the angle of the line of sight with the shower axis. The sonic field that is formed by the particle shower can be characterized as a thin, flat disk, with the shower axis forming the axis of the disk. For a typical hadronic shower, the sonic field is a disk of about 15 meter thick, while the radius of the disk can be as large as $1 \mathrm{~km}$. 


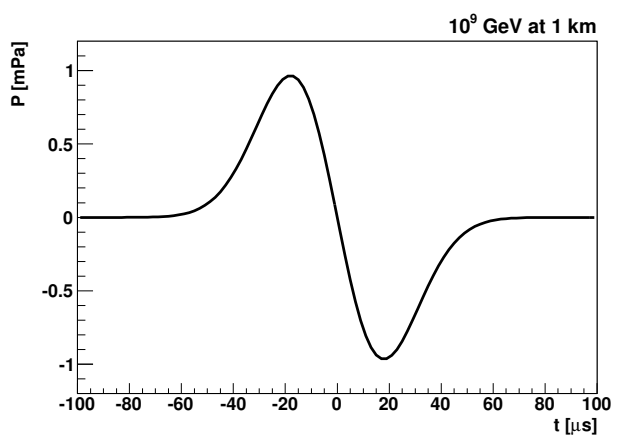

(a)

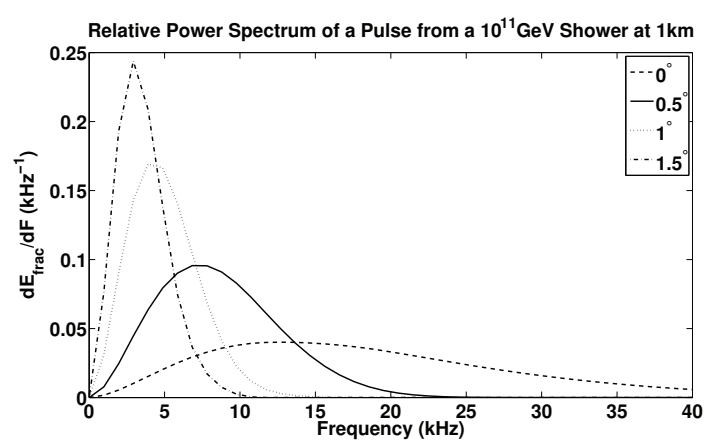

(b)

Figure 1: (a) Typical acoustic signal from a energetic neutrino induced particle shower. In (b) the relative power of a pulse as a function of the frequency. The spectrum is plotted for various angles w. r. t. to the plane perpendicular to the particle shower axis. Adapted from [10].

The absorption length of sea water in the frequency range of interest is relatively long: At the $25 \mathrm{kHz}$ the absorption length is about $1 \mathrm{~km}$, while at $10 \mathrm{kHz}$ it is almost $10 \mathrm{~km} \mathrm{[11].} \mathrm{These} \mathrm{values}$ for the absorption length indicate that indeed a kilometer sized detector would be possible. The concept of a deep sea acoustic neutrino telescope consists therefore of long strings, each equipped with several tens of hydrophones. A large number of strings would then form a fine grid of sensors with a total volume of many cubic kilometers. An optimized geometry of such a telescope is beyond the discussion of this paper. We mention, however, that the strings of hydrophones would consist of an oil filled hose to balance the isostatic pressure. The oil filled hose is further discussed in section 4 .

\subsection{Background noise}

Expected background noises are of several types. First of all, throughout the deep sea there is constant source of noise that is related to the state of the sea surface. White noise in the frequency range of interest is correlated to the rain and wave height at the sea surface. This relation of noise and sea state has been studied and modeled first by Knudsen [12]. In analogy to the Beaufort scale of wind force, the noise levels, called deep sea states have been parametrized. The lowest noise level, i. e. deep sea state zero (DDS0), is taken as a design value for the sensitivity of the hydrophone. At $10 \mathrm{kHz}$ the DSS0 has a power spectrum of $30 \mathrm{~dB}$ re $\mu \mathrm{Pa} / \sqrt{\mathrm{Hz}}$.

Noise sources of different nature may be transients noise sources. Potentially large source of background are the sound clicks of marine mammals and sperm whales in particular. Theses whales are found in every sea on Earth and produce transient signals in the range $5-35 \mathrm{kHz}[13,14]$. These signals, however, can be discriminated using the fact that these clicks come in a 'pulse train' (i. e. a repetitive structure of many individual clicks that are used for echolocation and communication). Moreover the sound level is much higher and the fact that the neutrino produces a (thin) line source (in contrast to a whale) gives rise to a distinct event topology and provides a handle to discriminate the two sources. 


\section{Fiber-based hydrophone system}

\subsection{Fiber based hydrophones}

Fiber optic sensors are well known in literature as a component for optical acoustic sensors. In this the core component is a fiber laser sensor acting as transducer for the acoustic pressure. Using a Fiber Bragg Grating (FBG) inside an (Erbium) doped fiber a laser system inside the core of the fiber is created. This technology is the so-called Distributed Feed Back fiber laser. Changes of the FBG length (due to pressure) results in wavelength shift of the laser (sensor).

At the position of the FBG the fiber is glued on a sensor (or mechanical transducer). In principle, many FBGs can inserted in a single fiber so that up to 20 sensors can be read out simultaneously. The sensor as developed at TNO is shown in figure 2. The photograph in the right panel shows the sensor as deployed in an oil filled hose.

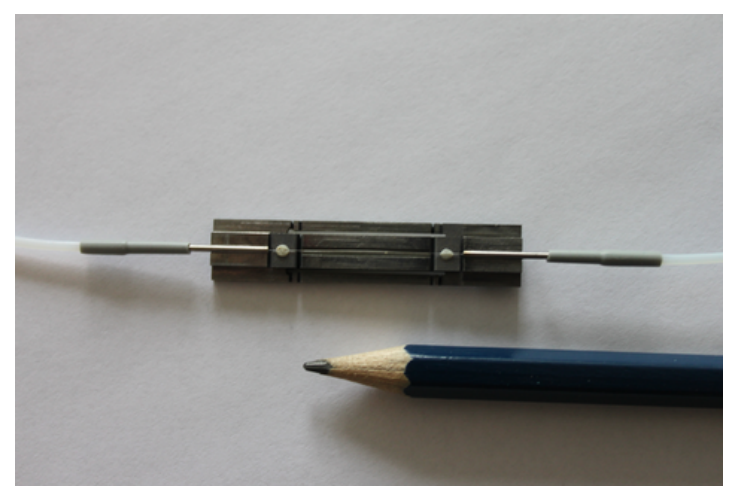

(a)

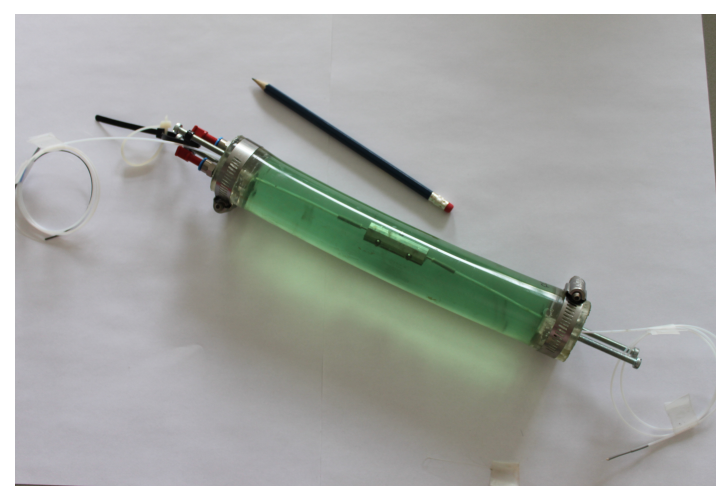

(b)

Figure 2: (a) Photograph of the hydrophone sensor as attached to an optical fiber. (Two glue dots can be seen). In (b) a photograph of the sensor in an oil hose is shown.

The principle of interrogation is depicted in figure 3. The wavelength shift that is induced by the strain in the cavity will be converted in to a phase shift using an interferometer. This interferometer is consists of a modified Mach-Zender interferometer that is designed to have a sensitivity (in combination with the sensor) of $-154 \mathrm{~dB}$ re $1 \mathrm{rad} / \mu \mathrm{Pa}$, normalized to 1 meter optical path difference. Phase shift in the light output of the interferometer will result in light intensity variation on to the photo-diodes. These photo-diodes are housed on custom made PCB boards that also include preamplifiers. Finally the signal is digitized and multiplexed using a 20 bit ADC and stored on to a PC. The sampling rate that was used during the measurements was $50 \mathrm{kHz}$.

The light source is a $980 \mathrm{~nm}$ pump laser. The pump laser is a commercial available laser which is set to produce a power of about $200 \mathrm{~mW}$. A narrow laser line width of about only $10 \mu \mathrm{W}$ is then emitted by the fiber laser.

\section{Hydrophones characterization}

\subsection{Experimental set up}

The hydrophones were characterized in an anechoic basin at TNO. This facility is dedicated 


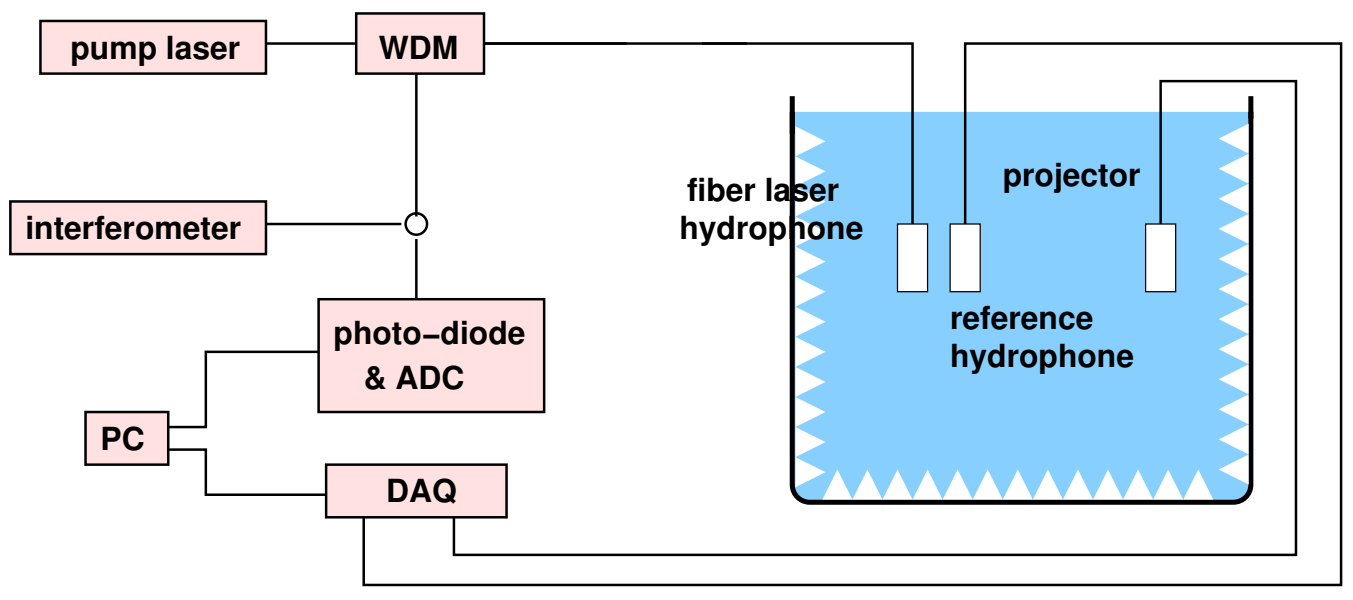

Figure 3: Schematic principle of an interrogator for the read out of a fiber laser hydrophone.

to underwater acoustics and it mainly consists of a large acoustically isolated basin of dimensions $10 \times 8 \times 8 \mathrm{~m}^{3}$. The experimental set-up is depicted in figure 3 .

Two identical fiber laser hydrophones were used in the experiments. One hydrophone was placed in the basin without any mechanical support or housing, while the second hydrophone was placed in a short oil-filled hose. The hose (as shown in Figure 2) has a diameter of $40 \mathrm{~mm}$ and is $240 \mathrm{~mm}$ in length. The oil that was used to fill the hose, was Shell Naturel HF-E 15. It has a natural viscosity of $14.9 \mathrm{~mm}^{2} / \mathrm{s}$ at $40^{\circ} \mathrm{C}$ and a density of $0.892 \mathrm{~kg} / \mathrm{l}$ at $20^{\circ} \mathrm{C}$.

Two commercial, pre-calibrated piezo-based hydrophones, from Brüel\&Kjaer, type B\&K 8101 and B\&K 8103 were used as references. In addition, two acoustic sources (also called projectors) have been used during the measurements, which each their specific operational acoustic frequency range. The projector for the measurements at lower frequencies was an Actran projector (type LFPX-4). A B\&K 8105 hydrophone was used as a projector for the higher frequencies. These projectors and its driver software allowed to generate several acoustic test signals, such as frequency sweeps or pure tones.

\subsection{Sensitivity and linearity}

Prior to the characterization of the hydrophone in the water basin, the self noise of the device was studied in a vibration isolated environment. Care was taken in the laboratory set-up to avoid as many noise sources as possible. In figure 4 we show the self noise of the laser fiber based hydrophone. At frequencies above $1 \mathrm{kHz}$, the noise floor of the laser fiber has a value of about 30 $\mathrm{dB} 1 \mathrm{re} \mu \mathrm{Pa}^{2} / \mathrm{Hz}$.

In figure 5 the response of the fiber laser hydrophone is shown compared to the reference hydrophone. The plot shows a flat response curve in the range of interest. A small increase in the responsivity of the fiber laser was found around $6 \mathrm{kHz}$. To determine the linearity of the hydrophone, a single tone was generated at a frequency of $10 \mathrm{kHz}$. The input to the projector was registered as being the input voltage to generate the sound and is shown in the right panel in figure 5. The figure shows a linear behavior of the hydrophones under test. All hydrophones are linear 


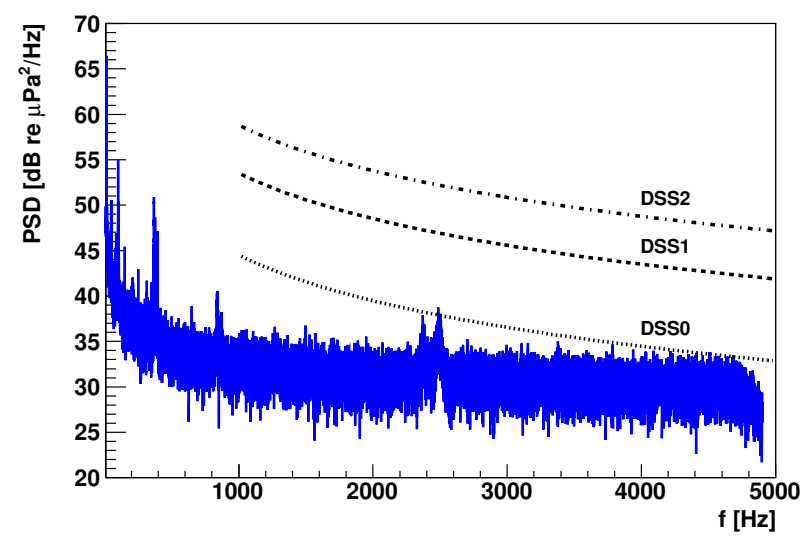

Figure 4: Self noise of the laser fiber based hydrophones.

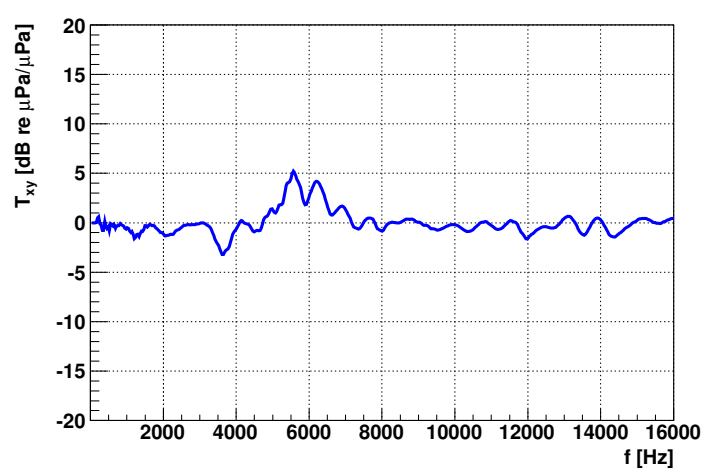

(a)

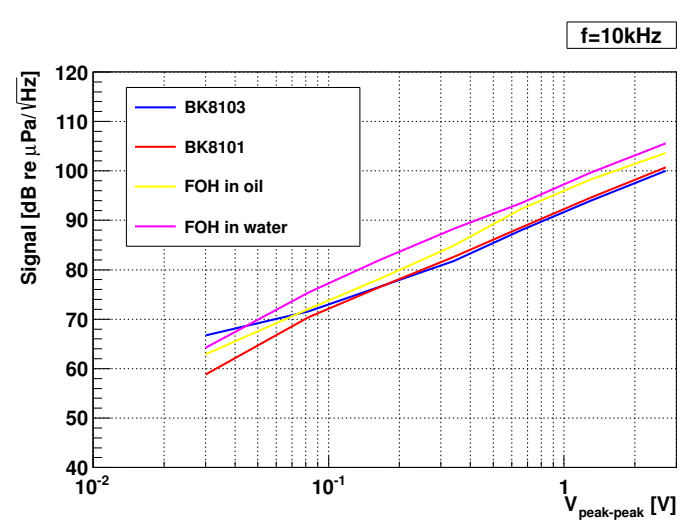

(b)

Figure 5: (a) Response of the fiber optic hydrophone (in oil) compared to the reference hydrophone. In (b) the linearity of several hydrophones as a function of the input voltage of the projector is plotted.

down to low levels to which the linearity of the projector was assured. One reference hydrophone (the B\&K 8103), however, showed some non-linearity due to self-noise of the device.

\subsection{Pulse reconstruction}

To simulate the pulse that can be expected from a cosmic ray, a large number of bipolar pulses were generated and stored in a sound file. This sound file was played underwater. It was made sure that the pulse had the amplitude and duration according to the simulated pulses that have been recorded in literature [10]. The height of the pulse was verified using the calibrated B\&K (type 8101) hydrophone.

In figure 6 (a) an example of a train of recorded pulses is shown for the fiber laser hydrophone and the reference hydrophone. Here, only a simple pass-band filter between 5 and $7 \mathrm{kHz}$ was 
applied (i.e. 4th order Butterworth filter) to pronounce the peaks. For both hydrophones the peaks clearly stand out of the background. If we zoom in on a single pulse as shown in figure 6 (b), we

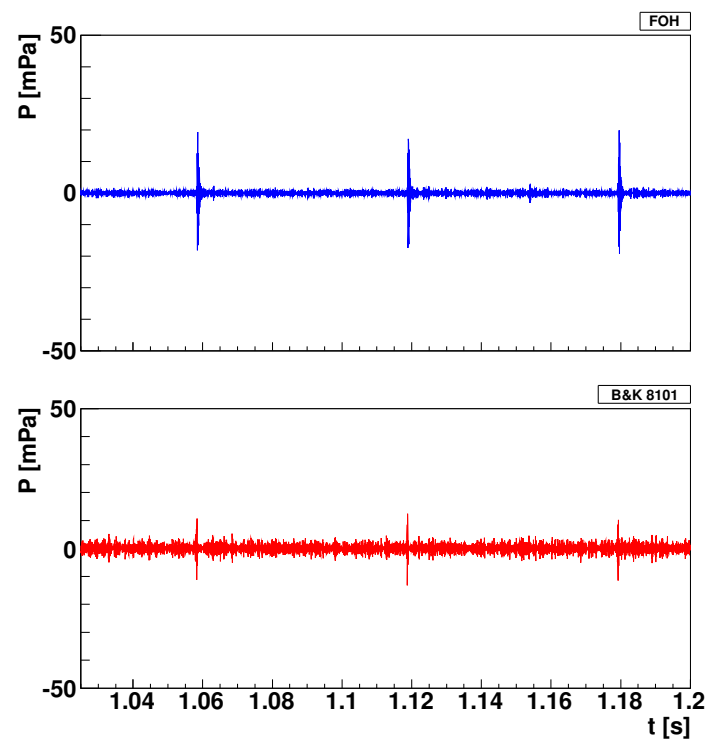

(a)

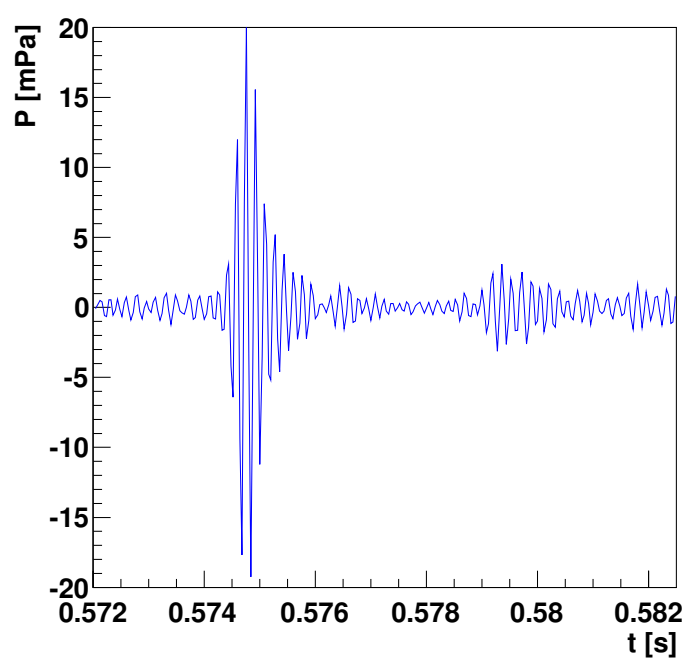

(b)

Figure 6: (a) Time trace of both the fiber laser hydrophone (upper panel) as well as the $\mathrm{B} \& \mathrm{~K}$ hydrophone (lower panel). In (b) an example of a single pulse is shown. The pulse has been recorded using the fiber laser hydrophone. Next to the recorded pulse also the echo can be seen with an amplitude of only several $\mathrm{mPa}$.

see that the fiber laser hydrophone shows some ringing at the tail of the pulse. The origin of the ringing is yet unknown.

\section{Conclusions}

Acoustic detection of cosmic rays in water is a promising means to study the high-end of the energy spectrum of cosmic neutrinos. The origin of the most energetic neutrinos is still a mystery and is a subject of investigation as well as a possible signature of the GZK cut-off limit in the neutrino spectrum. To detect the high energy cosmic neutrinos poses a challenge, due to the low expected flux and the low interaction cross-section of neutrinos. Therefore, the experimental set up that is used to study the cosmic neutrinos is required to have a large sensitive volume. One way to meet this requirement is to utilize the fact that particle showers induce a thermo-acoustic signal in water, which can be detected using hydrophones.

A hydrophone detection system based of fiber laser optics has been designed and realized. The benefits of this technology are that the sensors are cost effective and so that a large volume can be equipped with a large number of sensors. Secondly, the sensors are straightforward to implement in long strings and require a minimum of front-end electronics. Finally and most important, the optical fiber technology allows to construct an extremely sensitive sensor.

To investigate the suitability of an application in a cosmic ray set-up, a measurement campaign was conducted to characterize the hydrophones. It has been found that fiber laser hydrophones 
exhibit a low self-noise level, which corresponds to level that is comparable to the lowest level of ambient noise in the ocean, the so-called deep sea state zero. Hence a hydrophone system could be constructed that is ocean noise limited. This conclusion perfectly fits the motto of the TIPP conference: "Instrumentation as enabler of Science".

\section{References}

[1] G. A. Askaryan, Acoustic recording of neutrinos, Zemlia i Vselennaia, 1 (1979) 13

[2] J. G. Learned, Acoustic radiation by charged atomic particles in liquids: An analysis Phys. Rev. D 19 (1979) 3293

[3] IceCube collaboration, Evidence for High-Energy Extraterrestrial Neutrinos at the IceCube Detector, Science 342 (2013) 1242856,

IceCube collaboration, Observation of High-Energy Astrophysical Neutrinos in Three Years of IceCube Data, arXiv:1405.5303

[4] K. Greisen, Phys. Rev. Lett., End to the Cosmic-Ray Spectrum?, Phys. Rev. Lett., 16 (1966) 748 ; G. T. Zatsepin and V. A. Kuzmin, Upper Limit of the Spectrum of Cosmic Rays, JETP Lett., 4 (1966) 78.

[5] R. U. Abbasi et al (The High Resolution Fly's Eye Collaboration), First Observation of the Greisen-Zatsepin-Kuzmin Suppression, Phys. Rev. Lett., 100-10 (2008) 101101.

[6] J. Abraham et al (The Pierre Auger Collaboration), Observation of the Suppression of the Flux of Cosmic Rays above $4 \times 10^{19} \mathrm{eV}$, Phys. Rev. Lett., 101-6 (2008) 061101.

[7] E. Waxmann, IceCube's neutrinos: The beginning of extra-Galactic neutrino astrophysics?, arXiv:1312.0558v1

[8] G. A. Askaryan, Hydrodynamic Radiation From the Tracks of Ionizing Particles in Stable Liquids, Sov. J. At. En. 3 (1957) 921, Russian original: At. Energ. 3 (1957) 152

[9] L. Sulak et al., Experimental studies of the acoustic signature of proton beams traversing fluid media, Nucl. Inst. and Meth. 161 (1979) 203.

[10] R. Lahmann, Ultra-High-Energy Neutrinos and Their Acoustic Detection in the Sea, Habilitation Thesis, Friedrich-Alexander Universität Erlangen (2011)

[11] M. A. Ainslie, J. G. McColm, A simplified formula for viscous and chemical absorption in sea water, J. Acoustical Society of America, 103(3) (1998) 1671-1672.

[12] V. O. Knudsen, R. S. Alford, and J. W. Emling, Underwater ambient noise, J. Mar. Res. 3 (1948) 410

[13] A. M. von Benda-Beckmann et al., Effect of towed array stability on instantaneous localization of marine mammals, J. Acoust. Soc. Am. 134 (2013) 2409

[14] P. T Madsen et al., Sperm whale sound production studied with ultrasound time/depth-recording tags, J. Exp. Biology 205 (2002) 1899 